\title{
ZEROS OF ENTIRE FUNCTIONS IN SEVERAL COMPLEX VARIABLES
}

\author{
BY \\ RICHARD A. KRAMER( $\left.{ }^{1}\right)$
}

\begin{abstract}
A geometric condition on the zero set of an entire function $f$ in $C^{N}(N \geq 1)$ is presented which is both necessary and sufficient for $f$ to have the same zeros as some polynomial in $C^{N}$.
\end{abstract}

1.

1.1. Introduction. Let $f$ be an entire function in $\mathrm{C}^{N}(N \geq 1)$. It is natural to seek a geometric criterion for the zero set, $Z(f)$, of $f$ that is both necessary and sufficient for the existence of a polynomial $P$ such that both $f / P$ and $P / f$ are zero free entire functions. We will say, in this case, that $f$ and $P$ have the same zeros. Such a criterion was originated by Walter Rudin; see [2]. In $\$ 2$, we will use methods similar to those he employed to show that $f$ has the zeros of a polynomial if and only if the intersection of $Z(f)$ with

$$
\Delta_{r}=\left\{z \in \mathbf{C}^{N}: r_{1}\left|z_{1}\right|=\cdots=r_{N}\left|z_{N}\right|\right\}
$$

is compact for some choice of positive constants $r_{i}, i=1, \ldots, N$. This result will be extended in $\$ 3$.

The following notation will be used throughout. $T^{N}$ will denote the Cartesian product of $N$ copies of the unit circle $T$, and $r T$ will designate the circle of radius $r>0$ with center at the origin. The multi-index $j=\left(j_{1}, \cdots, j_{N}\right)$, where $j_{1}, \cdots, j_{N}$ are integers, will be used with $|j|=j_{1}+\cdots+j_{N}$, and $w^{j}=w_{1}^{j_{1}} \cdots w_{N}^{j_{N}}$ for $w \in \mathbf{C}^{N}$. If $f$ is an entire function in $\mathbf{C}^{N}$ and $\alpha \in \mathbf{C}^{K}$, $K<N$, then $f_{a}$ is the entire function defined on $\mathrm{C}^{N-K}$ by

$$
f_{\alpha}(w)=f\left(w_{1}, \alpha_{1} w_{1}, \ldots, \alpha_{K} w_{1}, w_{2}, \ldots, w_{N-K}\right) .
$$

$D_{i}$ will designate differentiation with respect to the $i$ th coordinate.

A wedge $W$ in $\mathbf{C}^{N}(N \geq 1)$ is any subset of the form

$$
W=\left\{z \in \mathbf{C}^{N}\left|r_{i}\right| z_{1}|\leq| z_{i}\left|\leq s_{i}\right| z_{1}|, i=2, \cdots, N, K \leq| z_{1} \mid\right\}
$$

Received by the editors August 30, 1971 and, in revised form, December 1, 1971 . AMS (MOS) subject classifications (1969). Primary 3205; Secondary 3244.

Key words and phrases. Polynomial, entire function, zeros of an entire function.

(1) This paper presents part of the author's doctoral dissertation which was written at the University of Wisconsin under the guidance of Professor Walter Rudin. 
where $r_{i}<s_{i}, i=2, \cdots, N$, and $K<\infty$. When $N=1$, a wedge is the complement, in $\mathrm{C}$, of an open disc with center at the origin.

1.2. Proposition. Let $Q$ be an annular neighborbood of $T$ in $\mathrm{C}$, and consider

$$
P(w, \alpha)=\sum_{0 \leqslant|j| \leqslant n} b_{j}(\alpha) w^{j}, \quad w \in \mathbf{C}^{N}(N \geq 1), \alpha \in Q
$$

where the coefficients $b_{j}$ are bolomorpbic in $Q$. Then there is a circle $r T \subset Q$ and a wedge $W \subset C^{N}$ sucb that $Z(P) \cap(W \times r T)=\varnothing$.

Proof. The zeros of a monic polynomial in one variable are bounded in modulus by the sum of the absolute values of the coefficients. When $N=1$, therefore, the zeros of $P(\cdot, \alpha)$ are uniformly bounded in modulus for all $a \in r T \subset Q$ provided that the leading coefficient has no zeros on that circle; since the coefficients are holomorphic in $Q$, there are many such circles.

For $N \geq 2$, write $P$ in the form

$$
P_{\beta}(\lambda, \alpha)=\sum_{i=0}^{m} H_{i}(\beta, \alpha) \lambda^{i}, \quad \alpha \in Q, \beta \in \mathbf{C}^{N-1}, \lambda \in \mathbb{C}^{1},
$$

where $H_{m} \equiv 0$. Assume that there is a wedge $W^{\prime} \subset \mathrm{C}^{N-1}$ and a circle $r T \subset Q$ such that $Z\left(H_{m}\right) \cap\left(W^{\prime} \times r T\right)=\emptyset$. Choose positive constants $r<s$ such that $E=\left\{\beta \in W^{\prime}: s \leq\left|\beta_{1}\right| \leq r\right\}$ is a nonempty compact subset of $\mathrm{C}^{N-1}$. Then, as before, the zeros of $P_{\beta}(\cdot, \alpha)$ are uniformly bounded for all $\alpha \in r T$ and $\beta \in E$; for the wedge $W=\left\{(\lambda, \beta \lambda) \in \mathbf{C}^{N}: \beta \in E, K \leq|\lambda|\right\}$, with $K$ suitably large, we have, therefore, $Z(P) \cap(W \times r T)=\emptyset$.

1.3. Remark. Let $W$ be a wedge in $\mathrm{C}^{N}(N \geq 2)$. There is a nonsingular linear transformation $\Theta$ and a positive constant $A$ such that the complement of $\Theta(W)$ is contained in the subset of $\mathbf{C}^{N}$ defined by $\left|w_{1}\right|<A\left(1+\left|w_{2}\right|+\cdots+\left|w_{N}\right|\right)$.

Proof. It is sufficient to show this for the symmetric case where $s_{i}=1 / r_{i}$ and $0<r_{i}<1, i=2, \cdots, N$.

Put

$$
\begin{gathered}
z_{1}=w_{1}, \\
z_{2}=w_{1}+w_{2}, \\
\cdot \\
\cdot \\
\cdot \\
z_{N}=w_{1}+w_{N},
\end{gathered}
$$

then 


$$
\begin{aligned}
w_{1} & =z_{1}, \\
w_{2} & =z_{2}-z_{1}, \\
& \cdot \\
& \cdot \\
w_{N} & =z_{N}-z_{1}
\end{aligned}
$$

defines a nonsingular linear transformation $\Theta$.

If $\left|z_{1}\right|<r_{i}\left|z_{i}\right|, i=2, \cdots, N$, then $\left|w_{1}\right|=\left|z_{1}\right|<r_{i}\left|z_{i}\right| \leq r_{i}\left(\left|w_{1}\right|+\left|w_{i}\right|\right)$; therefore, $\left|w_{1}\right|<\left(r_{i} /\left(1-r_{i}\right)\right)\left|w_{i}\right|$. And if $\left|z_{i}\right|<r_{i}\left|z_{1}\right|, i=2, \cdots, N$, then $\left|w_{1}\right| \leq\left|z_{i}\right|+\left|w_{i}\right|<r_{i}\left|z_{1}\right|+\left|w_{i}\right|=r_{i}\left|w_{1}\right|+\left|w_{i}\right|$; therefore $\left|w_{1}\right|<\left(1 /\left(1-r_{i}\right)\right)\left|w_{i}\right| \cdot$ Thus there exists a positive constant $A$ such that the complement of $\Theta(W)$ is contained in the region defined by $\left|w_{1}\right|<A\left(1+\left|w_{2}\right|+\cdots+\left|w_{N}\right|\right)$.

1.4. Remark. Let $H$ be a compact, connected, locally connected subset of $\mathrm{C}^{N}(N \geq 1)$, and assume that $f \not 0$ is holomorphic in a neighborhood of $\mathrm{C} \times H$. Then

(a) the zeros of $f(\cdot, \alpha)$ are bounded in modulus uniformly for $\alpha \in H$ if and only if $f(\cdot, a)$ has the same number of zeros for each $\alpha \in H$.

(b) Assuming $H$ has at least two distinct points, let $E=\{\alpha \in H: f(\cdot, \alpha) \equiv$ $0\}$, and suppose there is a constant $M<\infty$ such that the set, $S$, of all points $\alpha \in H-E$ for which the zeros of $f(\cdot, \alpha)$ are not bounded in modulus by $M$ is countable, then $S$ is empty.

Proof. (a) If $f(\lambda, \alpha) \neq 0$ for all $|\lambda| \geq r$ and all $\alpha \in H$, then the number of zeros of $f(\cdot, \alpha)$ is given by

$$
N(\alpha)=\frac{1}{2 \pi i} \int_{|\xi|=r} \frac{\mathscr{D}_{1} f(\xi, \alpha)}{f(\xi, \alpha)} d \xi, \quad \alpha \in H,
$$

which is continuous, integer valued, and, therefore, constant on the connected set $H$.

Conversely assume $f(\cdot, \alpha)$ has $m$ zeros, counted according to multiplicities, for each $\alpha \in H$. For each $r>0$ and each $\alpha \in H$, define

$$
N_{r}(\alpha)=\frac{1}{2 \pi i} \int_{|\xi|=r} \frac{\mathscr{D}_{1} f(\xi, \alpha)}{f(\xi, \alpha)} d \xi,
$$

provided that $f(\xi, \alpha) \neq 0$ for all $\xi \in r T$. Then $N_{r}(\alpha)$ is the number of zeros of $f(\cdot, \alpha)$ with modulus less than $r$. For fixed $r, N_{r}(\cdot)$ is continuous in some neighborhood of $\alpha$. For each $\alpha \in H$, choose $r_{a}>0$ such that $N_{r_{a}}(\cdot) \equiv m$ on some neighborhood $\mathcal{O}_{a}$ of $a$. Since $H$ is compact, finitely many of these neighborhoods, $\mathcal{O}_{a_{1}}, \cdots, \mathcal{O}_{a_{n}}$, cover it; and, therefore, the zeros of $f(\cdot, \alpha)$ are bounded uniformly in modulus by $r=\max \left\{r_{a_{1}}, \cdots, r_{a_{n}}\right\}$ for all $\alpha \in H$. 
(b) Let $\alpha^{\prime} \in H-E$ and suppose $f_{\alpha^{\prime}}$ has no zeros on the circle $r T$. Let $D$ be any neighborhood of $w^{\prime}$ such that, for each $\alpha \in(H-E) \cap D, f(\cdot, \alpha)$ has no zeros on $r T$ and $(H-E) \cap D$ is connected. The number of zeros of $f(\cdot, \alpha)$ with modulus less than $r$ is given by

$$
N(\alpha)=\frac{1}{2 \pi i} \int_{|\lambda|=r} \frac{\mathscr{D}_{1} f(\lambda, \alpha)}{f(\lambda, \alpha)} d \lambda, \quad \alpha \in(H-E) \cap D .
$$

Since $(H-E) \cap D$ is connected and $N$ is continuous, $f(\cdot, \alpha)$ has the same number of zeros with modulus less than $r$ for each $\alpha \in(H-E) \cap D$. It follows, because $H-(S \cup E)$ is dense in $H-E$, that $\alpha^{\prime} \notin S$. This concludes the proof.

2.

2.1. Theorem. If $f$ is an entire function in $C^{N}(N \geq 1)$, not identically zero, then $f=P \cdot \exp b$, where $P$ is a polynomial and $b$ is entire, if and only if the intersection of $Z(f)$ with some $\Delta_{r}$ is compact.

The necessity portion of this theorem is an immediate consequence of Proposition 1.2. Several lemmas precede the proof of sufficiency. The first is essentially a proof that Walter Rudin gave in [2]. The next two lemmas deal with the two variable case and the last two are the induction step. Lemma 2.6 will be used again in \$3; for this reason it and the other lemmas appear in a more general form than is required to prove the sufficiency statement. Several corollaries of Theorem 2.1 appear at the end of this section.

A subset $H$ of $\mathrm{C}^{N}(N \geq 1)$ will be called a local determining set when it has the property: for each $\alpha \in H$ and each polydisc $D$ centered at $\alpha, H \cap D$ is a determining set for the holomorphic functions on $D$; that is, if $f$ is holomorphic on $D$ and identically zero on $H \cap D$, then in fact $f$ is identically zero on all of $D$. It is easy to see that the finite Cartesian product of local determining sets is again a local determining set.

2.2. Lemma. Assume $f$ is bolomorphic in a neighborbood of $\mathbf{C}^{N} \times H(N \geq 2)$, where $H$ is a compact, connected subset of $\mathbf{C}^{K}(K \geq 1)$, which is a determining set for the bolomorphic functions on each of its connected neighborboods. If there is a wedge $W$ in $\mathbf{C}^{N}$ such that $Z(f) \cap(W \times H)=\varnothing$, then there is a neighborbood, $\mathcal{O}$, of $H$ in $\mathrm{C}^{K}$ and

$$
\Lambda(z, \alpha)=\sum_{0 \leqslant|j| \leqslant n} b_{j}(\alpha) z^{j}, \quad(z, \alpha) \in \mathbf{C}^{N} \times \mathcal{O},
$$

where each of the coefficients $b_{j}$ is bolomorpbic in $\mathcal{O}$ and such that, for each $a \in H$, the polynomial $\Lambda(\cdot, \alpha)$ and the entire function $f(\cdot, \alpha)$ bave the same zeros in $\mathbf{C}^{N}$; that is, botb $f(\cdot, \alpha) / \Lambda(\cdot, \alpha)$ and $\Lambda(\cdot, \alpha) / f(\cdot, \alpha)$ are zero free entire functions. 
Proof. Remark 1.3 shows that there is a nonsingular linear transformation

$$
\Theta:\left\{\begin{array}{rl}
z_{1} & =\theta_{1}(t), \\
z_{2} & =\theta_{2}(t), \\
& \cdot \\
& \cdot \\
z_{N} & =\theta_{N}(t),
\end{array} t=\left(t_{1}, \cdots, t_{N}\right) \in \mathbf{C}^{N},\right.
$$

and a positive constant $A$ such that, if we put $g(t, \alpha)=f\left(\theta_{1}(t), \cdots, \theta_{N}(t), \alpha\right)$, then for each $\alpha \in H$,

$$
Z(g(\cdot, \alpha)) \subset\left\{t \in \mathbb{C}^{N}:\left|t_{N}\right|<A\left(1+\left|t_{1}\right|+\cdots+\left|t_{N-1}\right|\right)\right\} .
$$

Set $\tilde{t}=\left(t_{1}, \cdots, t_{N-1}\right)$ and $t=\left(\tilde{t}, t_{N}\right)$. To each $r>A$ there corresponds a connected neighborhood, $\mathcal{O}_{r}$, of $H$ in $\mathbf{C}^{K}$ such that $g$ has no zeros on

$$
\left\{t \in \mathbf{C}^{N}: A\left(1+\left|t_{1}\right|+\cdots+\left|t_{N-1}\right|\right) \leq\left|t_{N}\right| \leq r\right\} \times \overrightarrow{\hat{O}_{r}} \text {. }
$$

Set

$$
\mathcal{U}_{r}=\left\{\tilde{t} . \in \mathbf{C}^{N-1}: A\left(1+\left|t_{1}\right|+\cdots+\left|t_{N-1}\right|\right)<r\right\} .
$$

For $(\tilde{t}, \alpha) \in \mathcal{U}_{r} \times \mathcal{O}_{r}$ and $p$ a nonnegative integer, define $\psi_{p}$ by

$$
\psi_{p}(\tilde{t}, \alpha)=\frac{1}{2 \pi i} \int_{|\xi|=r} \frac{\mathfrak{D}_{N} g}{g}(\tilde{t}, \xi, \alpha) \xi^{p} d \xi,
$$

where $\mathscr{D}_{N}$ denotes differentiation with respect to the $N$ th coordinate $\xi$. If $r_{1}<r_{2}$, then, for $(\tilde{t}, a) \in\left(\mathcal{U}_{r_{1}} \times \mathcal{O}_{r_{1}}\right) \cap\left(\mathcal{U}_{r_{2}} \times \mathcal{O}_{r_{2}}\right)$, the path of integation in (1) can be moved to any circle with center at the origin and radius between $r_{1}$ and $r_{2}$ without changing the value of the integral. Thus the two functions given by (1) agree on $\mathcal{U}_{r_{1}} \times\left(\mathcal{O}_{r_{1}} \cap \mathcal{\Theta}_{r_{2}}\right)$; therefore each function $\psi_{p}, p=0,1,2, \ldots$, is well defined and holomorphic on

$$
\Omega=\bigcup_{r>A} U_{r} \times \Theta_{r}
$$

For each $(\tilde{t}, a) \in \Omega, \psi_{0}(\tilde{t}, a)$ is the number of zeros of $g(\tilde{t}, \cdot, a)$ with modulus less than $A\left(1+\left|t_{1}\right|+\cdots+\left|t_{N-1}\right|\right)$; hence $\psi_{0}$ is constant on the connected set $\Omega$ since it is continuous and integer valued there. Call this constant $m$; then $m$ is a nonnegative integer. If $m=0$, then for each $\alpha \in H$, $g(\cdot, \alpha)$ is zero free, and $\Lambda \equiv 1$ satisfies the ccaclusion of the theorem.

Assume $m>0$. For $(\tilde{t}, a) \in \Omega$, let $\zeta_{1}(\tilde{t}, a), \cdots, \zeta_{m}(\tilde{t}, a)$ be the zeros of $g(\tilde{t}, \cdot, a)$, counted according to multiplicity, whose absolute value is less than 
$A\left(1+\left|t_{1}\right|+\cdots+\left|t_{N-1}\right|\right)$. When $\alpha \in H$ these are all of the zeros of the entire function $g(\tilde{t}, \cdot, \alpha)$. The residue theorem shows that the integral in (1) is just

$$
\psi_{p}(\tilde{t}, \alpha)=\sum_{i=1}^{m} \zeta_{i}^{p}(\tilde{t}, \alpha), \quad(\tilde{t}, \alpha) \in \Omega .
$$

Define

$$
P\left(\tilde{t}, t_{N}, \alpha\right)=\prod_{i=1}^{m}\left(t_{N}-\zeta_{i}(\tilde{t}, \alpha)\right)=t_{N}^{m}+\sum_{i=1}^{m}(-1)^{i} \sigma_{i}(\tilde{t}, \alpha) t_{N}^{m-i},
$$

whenever $(\tilde{t}, \alpha) \in \Omega$ and $t_{N} \in \mathbf{C}$. Each coefficient $\sigma_{i}$ is the symmetric function on $\zeta_{1}, \ldots, \zeta_{m}$ taken $i$ at a time. Newton's identities say that the symmetric functions $\sigma_{i}$ and the power sums $\psi_{i}$ are related by

$$
-i(-1)^{i} \sigma_{i}=\psi_{i}+(-1)^{1} \psi_{i-1} \sigma_{1}+\cdots+(-1)^{i-1} \psi_{1} \sigma_{i-1}, \quad 1 \leq i \leq m,
$$

which shows, successively, that the coefficients $\sigma_{i}$ are holomorphic in $\Omega$. In particular, for each $\alpha \in H, \sigma_{i}(\cdot, \alpha)$ is an entire function in $\mathbf{C}^{N}$. Moreover, $\left|\zeta_{i}(\tilde{t}, a)\right|<A\left(1+\left|t_{1}\right|+\cdots+\left|t_{N-1}\right|\right)$, for all $(\tilde{t}, \alpha) \in \Omega$. Thus, for $\alpha \in H$, each $\sigma_{i}(\cdot, a)$ is an entire function in $\mathrm{C}^{N}$ with

$$
\left|\sigma_{i}(\cdot, \alpha)\right|<\left(\begin{array}{c}
m \\
i
\end{array}\right)\left[A\left(1+\left|t_{1}\right|+\cdots+\left|t_{N-1}\right|\right)\right]^{i},
$$

and this implies that each $\sigma_{i}(\cdot, \alpha)$ is actually a polynomial in $\mathrm{C}^{N-1}$ whenever $\alpha \in H$.

Let $D_{r}=D(0,(1 /(N-1))[r / A-1])$ be the open polydisc in $C^{N-1}$ with center at the origin and radius $(1 /(N-1))[r / A-1]$ in each coordinate. This

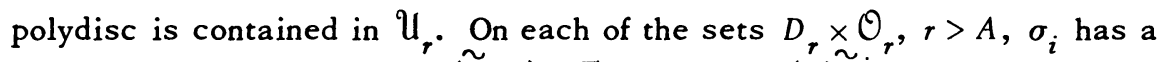
representation of the form $\sigma_{i}(\tilde{t}, a)=\Sigma_{0 \leq|j|<\infty} \phi_{i, j}(\tilde{a}) \tilde{t}^{j}$, where the coefficients $\phi_{i, j}$ are holomorphic in $\mathcal{O}_{r}$ and independent of $r$. Thus this representation holds on $U_{r>A}\left(D_{r} \times \mathcal{O}_{r}\right) \subset \Omega$. But (4) implies that $\sigma_{i}(\tilde{t}, \alpha)=\Sigma_{0 \leq|j| \leq{ }_{i}} \phi_{i, j}(\alpha) \tilde{t}^{j}$, whenever $a \in H$. In particular, the coefficients $\phi_{i, j}$ vanish identically on $H$ whenever any one of the indices $j_{1}, \ldots, j_{N-1}$ is bigger than $i$. Let $\mathcal{O}$ be a connected neighborhood of $H$ with $\mathcal{O}$ contained in some $\mathcal{O}_{r}, r>A$. By the hypothesis, $H$ is a determining set for the holomorphic functions on $\mathcal{O}$. Thus the coefficients $\phi_{i, j}$, which vanish identically on $H$, vanish throughout $\mathcal{O}$, and the representation $\sigma_{i}(\tilde{t}, \alpha)=\Sigma_{0 \leq|j| \leq i} \phi_{i, j}(\alpha)_{t}{ }^{j}$ is valid in $\mathrm{C}^{N-1} \times \mathcal{O} . P$, therefore, has the representation

$$
P\left(\tilde{t}, t_{N}, \alpha\right)=t_{N}^{m}+\sum_{i=1}^{m}(-1)^{i}\left(\sum_{0 \leqslant|j| \leqslant i} \phi_{i, j}(\alpha) \tilde{t}^{j}\right) t_{N}^{m-i},
$$

for $\left(\tilde{t}, t_{N}, \alpha\right) \in \mathbf{C}^{N} \times \mathcal{O}$. 
In the original coordinates, (5) becomes

$$
\Lambda(z, \alpha)=\sum_{0 \leqslant|j| \leqslant n} b_{j}(\alpha) z^{j}, \quad(z, \alpha) \in \mathbf{C}^{N} \times \mathcal{O},
$$

where each coefficient $b_{j}$ is holomorphic in $\mathcal{O}$, and the polynomial $\Lambda(\cdot, a)$ and the entire function $f(\cdot, \alpha)$ have the same zeros in $\mathbf{C}^{N}$ for each $\alpha \in H$.

2.3. Lemma. Let $H$ be a compact, connected subset of $\mathbf{C}^{K}(K \geq 1)$, and assume that $f$ is bolomorphic in a neigbborbood of $\left\{z \in \mathbf{C}^{2}:\left|z_{i}\right|>M, i=1,2\right\}$ $\times H$, for some constant $M<\infty$. If $Z(f) \cap(\Delta \times H)=\varnothing$, then to eacb $\alpha \in H$ corresponds a neighborbood $\mathcal{O}_{\alpha}$ of $\alpha$ in $\mathrm{C}^{K}$ and a wedge $W_{\alpha}$ in $\mathbf{C}^{2}$ such that $Z(f) \cap\left(W_{a} \times\left(\Theta_{a} \cap H\right)\right)=\varnothing$. Here, as well as elsewbere, $\Delta=\Delta_{(1, \cdots, 1)^{*}}$

Proof. To each $r>M$ corresponds $0<\epsilon_{r}$ such that $f$ has no zero on $\bar{Q}_{r} \times \bar{Q}_{r} \times \bar{A}_{\epsilon_{r}}$, where

$$
A_{\epsilon_{r}}=\left\{w \in \mathbf{C}^{K}: \text { distance }(w, H)<\epsilon_{r}\right\} \text { and } Q_{r}=\left\{\lambda \in \mathbf{C}: r-\epsilon_{r}<|\lambda|<r+\epsilon_{r}\right\} .
$$

For $\left(z_{1}, a\right) \in Q_{r} \times A_{\epsilon_{r}}$ and each nonnegative integer $p$, define

$$
\psi_{p}\left(z_{1}, \alpha\right)=\frac{1}{2 \pi i} \int_{|\xi|=r+\epsilon_{r}} \frac{\mathscr{D}_{2} f}{f}\left(z_{1}, \xi, \alpha\right) \xi^{p} d \xi
$$

where $\mathcal{D}_{2}$ denotes differentiation with repect to $\xi$. If $r_{1}<r_{2}$ are such that $\left(Q_{r_{1}} \times A_{\epsilon_{r_{1}}}\right) \cap\left(Q_{r_{2}} \times A_{\epsilon_{r_{2}}}\right) \neq \varnothing$, then for $\left(z_{1}, \alpha\right)$ in this intersection, the path of integration in (1) can be moved to any circle with center at the origin and radius between $r_{1}-\epsilon_{r_{1}}$ and $r_{2}+\epsilon_{r_{2}}$, without changing the value of the integral. It follows that the two functions given by (1) agree on the intersection; thus each $\psi_{p}$ is well defined and holomorphic in $\Omega=\bigcup_{r>M}\left(Q_{r} \times A_{\epsilon_{r}}\right)$.

For each $\left(z_{1}, \alpha\right) \in \Omega, \psi_{0}\left(z_{1}, \alpha\right)$ is the number of zeros of $f\left(z_{1}, ., \alpha\right)$ whose absolute value is less than $\left|z_{1}\right|$; hence $\psi_{0}$ is constant on the connected set $\Omega$, since it is continuous and integer valued there. Call this constant $m$. Then $m$ is a nonnegative integer. If $m=0$, then $f\left(z_{1}, z_{2}, a\right) \neq 0$ whenever $\left|z_{2}\right|<\left|z_{1}\right|, M<\left|z_{1}\right|$, and $\alpha \in H$.

Assume $m>0$. For $\left(z_{1}, \alpha\right) \in \Omega$, let $\zeta_{1}\left(z_{1}, \alpha\right), \ldots, \zeta_{m}\left(z_{1}, \alpha\right)$ denote the zeros of $f\left(z_{1}, \cdot, a\right)$ whose absolute value is less than $\left|z_{1}\right|$. Then the residue theorem implies that

$$
\psi_{p}\left(z_{1}, \alpha\right)=\sum_{i=1}^{m} \zeta_{i}^{p}\left(z_{1}, \alpha\right), \quad\left(z_{1}, \alpha\right) \in \Omega
$$

Define

$$
P\left(z_{1}, z_{2}, \alpha\right)=\sum_{i=1}^{m}\left(z_{2}-\zeta_{i}\left(z_{1}, \alpha\right)\right)=z_{2}^{m}+\sum_{i=1}^{m}(-1)^{i} \sigma_{i}\left(z_{1}, \alpha\right) z_{2}^{m-i},
$$


for $\left(z_{1}, \alpha\right) \in \Omega$ and $z_{2} \in \mathbf{C}$. Newton's identities show that each $\sigma_{i}$ is a polynomial in the power sums $\psi_{p}$ and hence holomorphic in $\Omega$. In particular, since $\sigma_{i}$ is holomorphic in $Q_{r} \times A_{\epsilon_{r}}$, there exist coefficients holomorphic in $A_{\epsilon_{r}}$ such that

$$
\sigma_{i}\left(z_{1}, \alpha\right)=\sum_{j=-\infty}^{\infty} \phi_{i, j}(\alpha) z_{1}^{j}, \quad\left(z_{1}, \alpha\right) \in Q_{r} \times A_{\epsilon_{r}} .
$$

Moreover, since the coefficients $\phi_{i, j}$ are uniquely determined and $\Omega$ is the connected union of the sets $Q_{r} \times A_{\epsilon_{r}}$, it follows that the representation holds throughout $\Omega$.

Since $\left|\zeta_{i}\left(z_{1}, a\right)\right|<\left|z_{1}\right|$, we have

$$
\left|\sigma_{i}\left(z_{1}, \alpha\right)\right|<\left(\begin{array}{c}
m \\
i
\end{array}\right)\left|z_{1}\right|^{i}, \quad(z, \alpha) \in \Omega
$$

thus for each $\alpha \in H, \sigma_{i}(\cdot, \alpha)$ has a pole of order at most $i$ at infinity. Hence $\phi_{i, i}(\alpha)=\lim _{\lambda \rightarrow \infty} \lambda^{-i} \sigma_{i}(\lambda, \alpha), \alpha \in H$, exists as a finite complex number. This gives

$$
\sigma_{i}\left(z_{1}, \alpha\right)=\phi_{i, i}(\alpha) z_{1}^{i}+\widetilde{\sigma}_{i}\left(z_{1}, \alpha\right), \quad \alpha \in H,
$$

with

$$
\left|\tilde{\sigma}_{i}\left(z_{1}, \alpha\right)\right|<c\left|z_{1}\right|^{i-1}, \quad \alpha \in H
$$

for some constant $c<\infty$ and all sufficiently large $\left|z_{1}\right|$.

Set

$$
Q(\lambda, \alpha)=\lambda^{m}+\sum_{i=1}^{m}(-1)^{i} \phi_{i, i}(\alpha) \lambda^{m-i} .
$$

Then substitution of (4) into (3) shows that

$$
\frac{P\left(z_{1}, z_{2}, \alpha\right)}{z_{1}^{m}}=Q\left(\frac{z_{2}}{z_{1}}, \alpha\right)+\sum_{i=1}^{m}(-1)^{i} \frac{\tilde{\sigma}_{i}(z, \alpha)}{z_{1}^{i}}\left(\frac{z_{2}}{z_{1}}\right)^{m-i}
$$

for $\alpha \in H$. Since $Q(\cdot, \alpha) \nRightarrow 0$ and the $\phi_{i, i}$ are continuous, corresponding to each $\alpha_{0} \in H$, there are constants $r^{\prime}, r^{\prime \prime}$ such that $0<r^{\prime}<r^{\prime \prime}<1$, and a neighborhood $\mathcal{O}_{a_{0}}$ of $a_{0}$ in $\mathbf{C}^{K}$ such that

$$
\eta=\operatorname{rinf}_{r^{\prime} \leqslant|\lambda| \leqslant r^{\prime \prime} ; \alpha \epsilon H \cap \theta_{a_{0}}}|Q(\lambda, \alpha)|>0 .
$$

If $r^{\prime} \leq\left|z_{2} / z_{1}\right| \leq r^{\prime \prime}$ and $\alpha \in H \cap \mathcal{O}_{a_{0}}$, it follows from (5) and (6) that 


$$
\left|\frac{P\left(z_{1}, z_{2}, \alpha\right)}{z_{1}^{m}}\right| \geq \eta-\frac{c}{\left|z_{1}\right|} \sum_{i=1}^{m}\left|\frac{z_{2}}{z_{1}}\right|^{m-i} \geq \eta-\frac{c}{\left|z_{1}\right|} \frac{1}{1-r^{\prime \prime}}>0
$$

provided $\left|z_{1}\right|$ is large enough. In particular, $P\left(z_{1}, z_{2}, a\right) \neq 0$ under these conditions. Since $f$ and $P$ have the same zeros in $\left\{z \in \mathbf{C}^{2}:\left|z_{2}\right|<\left|z_{1}\right|\right.$, $\left.M<\left|z_{1}\right|\right\} \times H$, this concludes the proof.

2.4. Lemma. Assume $f$ is bolomorpbic in a neighborbood of $\mathrm{C}^{2} \times H$, where $H$ is a compact, connected, locally connected, local determining set contained in $\mathrm{C}^{K}(K \geq 1)$. If $Z(f) \cap(\Delta \times H)$ is compact, then corresponding to each $\alpha_{0} \in H$, there is a neighborbood $\mathcal{O}_{a_{0}}$ of $\alpha_{0}$ in $\mathrm{C}^{K}$ and

$$
\Lambda\left(z_{1}, z_{2}, \alpha\right)=\sum_{j_{1} j_{2}=0}^{n} b_{j_{1}, j_{2}}(\alpha) z_{1}^{j_{1}} z_{2}^{j_{2}},
$$

where each of the coefficients $b_{j_{1}, j_{2}}$ is bolomorphic in $\mathcal{O}_{a_{0}}$, such that for each $\alpha \in H \cap \mathcal{O}_{\alpha_{0}}$, the polynomial $\Lambda\left(\stackrel{\cdot,, \alpha}{j_{1}}\right)$ and the entire function $f(\cdot, \alpha)$ bave the same zeros.

Proof. Lemma 2.3 implies that there is a neighborhood $\mathcal{O}_{a_{0}}$ of $a_{0}$ in $\mathbf{C}^{K}$ and a wedge $W_{a_{0}}$ in $\mathbf{C}^{2}$ such that $Z(f) \cap\left[W_{a_{0}} \times\left(H \cap \overline{\mathcal{O}}_{a_{0}}\right)\right]=\emptyset$. But, for $\mathcal{O}_{a_{0}}$ suitably small, $H \cap \overline{\mathcal{O}}_{a_{0}}$ is a compact, connected subset of $\mathbf{C}^{K}$ which is a determining set for the holomorphic functions on each of its connected neighborhoods; thus the proof is concluded by applying Lemma 2.2.

2.5. Lemma. Assume $f$ is bolomorpbic in a neighborbood of $\mathrm{C}^{N} \times H(N \geq 2)$, where $H$ is a compact, connected, locally connected, local determining set contained in $\mathbf{C}^{K}(K \geq 1)$. If $Z(f) \cap(\Delta \times H)$ is compact, then there is a wedge $W$ in $\mathbf{C}^{N}$, symmetric about $\Delta$, such that $Z(f) \cap(W \times H)=\emptyset$.

Proof. It is sufficient to prove the lemma when $N \geq 3$; for if $f$ satisfies the hypothesis with $N=2$, then $g$, defined by $g\left(w, z_{1}, z_{2}, z_{3}\right)=f\left(w, z_{1}, z_{2}\right)$, also satisfies the hypothesis with $N=3$; hence if there is a wedge $W$ in $\mathbf{C}^{3}$, symmetric about $\Delta$, such that $Z(g) \cap(W \times H)=\varnothing$, then setting $\widetilde{W}=\left\{z=\left(z_{1}, z_{2}\right)\right.$ $\left.\in \mathbf{C}^{2}:\left(z_{1}, z_{2}, z_{3}\right) \in W\right\}$ gives a wedge in $\mathbf{C}^{2}$, symmetric about $\Delta$, for which $Z(f) \cap(\widetilde{W} \times H)=\varnothing$.

Let $N \geq 3$, and assume there are constants $0 \leq r_{i} \leq 1, i=3, \cdots, N$, and $M<\infty$ such that $f\left(z_{1}, \cdots, z_{N}, \alpha\right) \neq 0$ whenever $r_{i}\left|z_{1}\right| \leq\left|z_{i}\right| \leq\left(1 / r_{i}\right)\left|z_{1}\right|$, $i=3, \cdots, N,\left|z_{1}\right|=\left|z_{2}\right|, M \leq\left|z_{1}\right|$ and $\alpha \in H$. We will show that, for a proper choice of $M<\infty$, there exists a constant $0<r_{2}<1$ such that $f\left(z_{1}, \ldots, z_{N}, a\right)$ $\neq 0$, whenever $r_{i}\left|z_{1}\right| \leq\left|z_{i}\right| \leq\left(1 / r_{i}\right)\left|z_{1}\right|, i=2, \cdots, N, M \leq\left|z_{1}\right|$ and $a \in H$. The importance lies in the fact that $r_{2}$ can be chosen less than 1 without changing 
$r_{i}, i=3, \cdots, N$, for, when each $r_{i}$ can be chosen so that it is less than 1 , this gives the desired wedge. Such a choice is possible using successive applications of the result for $r_{2}$. If $r_{2}, \cdots, r_{j}$ are each less than 1 , then upon interchanging the second and $j+1$ coordinates, the result shows that, for large enough $M<\infty$, $r_{j+1}$ can also be chosen less than 1 .

Let $\eta=\left(\eta_{1}, \cdots, \eta_{N-2}\right)$ and set $\tilde{H}=H \times A_{1} \times \cdots \times A_{N-2}$, where $A_{i}=$ $\left\{\lambda \in \mathrm{C}: r_{i+2} \leq|\lambda| \leq 1 / r_{i+2}\right\}, i=1, \cdots, N-2 . \widetilde{H}$ is a compact, connected, locally connected, local determining set because each of the factors in the Cartesian product is such a set. Put $\tilde{f}\left(t_{1}, t_{2}, a, \eta\right)=f\left(t_{1}, t_{2}, \eta_{1} t_{1}, \cdots, \eta_{N-2} t_{1}, \alpha\right)$. Then $\widetilde{f}$ is holomorphic in a neighborhood of $\mathrm{C}^{2} \times \widetilde{H}$, and $Z(\widetilde{f}) \cap(\Delta \times \widetilde{H})$ is compact. Thus $\widetilde{f}$ and $\widetilde{H}$ satisfy the hypothesis of Lemma 2.4. Set $\xi=(\alpha, \eta)$ and fix $\xi^{\prime}=\left(\alpha^{\prime}, \eta^{\prime}\right) \in \widetilde{H}$. By Lemma 2.4 , there exist a connected neighborhood $\mathcal{O}_{\xi^{\prime}}$ of $\xi^{\prime}$ in $\mathrm{C}^{K+N-2}$ and

$$
\Lambda\left(t_{1}, t_{2}, \xi\right)=\sum_{i_{1}, i_{2}=0}^{n} b_{i_{1}, i_{2}}(\xi) t_{1}{ }_{1}^{i_{1}} t_{2}^{i_{2}}, \quad\left(t_{1}, t_{2}, \xi\right) \in \mathbf{C}^{2} \times{\overline{\mathcal{O}_{\xi}}}_{\xi^{\prime}}
$$

with each $b_{i_{1}, i_{2}}$ holomorphic in a neighborhood of $\overline{\mathcal{O}}_{\xi^{\prime}}$, and such that $\Lambda(\cdot, \cdot, \xi)$ and $\tilde{f}(\cdot, \cdot, \xi)$ have the same zeros, for each $\xi \in \widetilde{H} \cap \overline{\mathcal{O}}_{\xi^{\prime}}$. Since $H$ is locally connected, we may assume that $\tilde{H} \cap \overline{\mathcal{O}}_{\xi^{\prime}}$ is connected. In particular, $\Lambda\left(t_{1}, t_{2}, \xi\right) \neq 0$ whenever $\left|t_{1}\right|=\left|t_{2}\right|>M$ and $\xi \in \widetilde{H} \cap \overline{\mathcal{O}}_{\xi^{\prime}}$; thus by Remark 1.4, the number of zeros of $\Lambda_{\beta}(\cdot, \xi)$ is the same for each $\xi \in \widetilde{H} \cap \bar{\Theta}_{\xi^{\prime}}$ and each $\beta \in T$. Writing

$$
\Lambda_{\beta}(\lambda, \xi)=\sum_{k=0}^{m} H_{k}(\beta, \xi) \lambda^{k},
$$

where $H_{m} \not \equiv 0$, we have $H_{m}(\beta, \xi) \neq 0$, for all $\beta \in T$ and all $\xi \in H \cap \overline{\mathcal{O}}_{\xi^{\prime}}$. Therefore there is a constant $0<r_{\xi^{\prime}}<1$ such that $H_{m}$ is bounded away from zero on the compact see $X_{\xi^{\prime}}=\left\{\beta \in \mathrm{C}: r_{\xi^{\prime}} \leq|\beta| \leq 1 / r_{\xi^{\prime}}\right\} \times\left(\widetilde{H} \cap \overline{\mathcal{O}}_{\xi^{\prime}}\right)$. For $(\beta, \xi) \in X_{\xi^{\prime}}$, write

$$
\Lambda_{\beta}(\lambda, \xi)=H_{m}(\beta, \xi)\left\{\lambda^{m}+\frac{H_{m-1}(\beta, \xi)}{H_{m}(\beta, \xi)} \lambda^{m-1}+\cdots+\frac{H_{0}(\beta, \xi)}{H_{m}(\beta, \xi)}\right\},
$$

and set

$$
c_{j}=\max _{X_{\xi^{\prime}}}\left|H_{j}(\beta, \xi) / H_{m}(\beta, \xi)\right|, \quad j=0,1, \ldots, m-1 .
$$

Since each $c_{j}$ is finite, $M_{\xi^{\prime}}=1+\sum_{j=0}^{m-1} c_{j}<\infty$. It follows that $\Lambda_{\beta}(\cdot, \xi) \neq 0$ whenever $|\lambda|>M_{\xi^{\prime}}$ and $(\beta, \xi) \in X_{\xi^{\prime}}$. Thus $\tilde{f}\left(t_{1}, t_{2}, \xi\right) \neq 0$, whenever $r_{\xi^{\prime}} \leq\left|t_{2} / t_{1}\right| \leq 1 / r_{\xi^{\prime}}, M_{\xi^{\prime}}<\left|t_{1}\right|$ and $\xi \in \widetilde{H} \cap \overline{\mathcal{O}}_{\xi^{\prime}}$. Since $\widetilde{H}$ is compact, for 
sufficiently large $M<\infty$, there exists $0<r_{2}<1$ such that $\left.\tilde{f}_{t_{1}}, t_{2}, \xi\right) \neq 0$ whenever $r_{2}\left|t_{1}\right| \leq\left|t_{2}\right| \leq\left(1 / r_{2}\right)\left|t_{1}\right|, M<\left|t_{1}\right|$ and $\xi \in \widetilde{H}$. This concludes the proof.

2.6. Lemma. Assume $f$ is bolomorpbic in a neighborbood of $\mathrm{C}^{N} \times H(N \geq 2)$, where $H$ is a compact, connected, locally connected, local determining set contained in $\mathrm{C}^{K}(K \geq 1)$. If $Z(f) \cap(\Delta \times H)=\varnothing$, then there is a neighborbood $\mathcal{O}$ of $H$ in $\mathbf{C}^{K}$ and $\Lambda(z, \alpha)=\Sigma_{0} \leq|j| \leq n_{n} b_{j}(\alpha) z^{j}$, where each of the coefficients $b_{j}$ is bolomorphic in $\mathcal{O}$, such that for eacb $\alpha \in H$, the polynomial $\Lambda(\cdot, a)$ and the entire function $f(\cdot, a)$ bave the same zeros in $\mathbf{C}^{N}$.

Proof. The proof follows immediately from Lemmas 2.2 and 2.5.

2.7. Proof of the sufficiency portion of Theorem 2.1. The proof is well known when $N=1$. Assume $N \geq 2$, and that $\Delta_{r}=\Delta$. Set $\widetilde{f}(z, \alpha)=f(z)$, $z \in \mathbf{C}^{N}, \alpha \in \mathbf{C}$, and let $H$ be the unit circle in $\mathbf{C}$. Then Lemma 2.6 implies the existence of the polynomial $P(z)=\Lambda(z, 1)=\Sigma_{0 \leq|j| \leq n} b_{j}(1) z^{j}$ with the same zeros as $f$ in $\mathbf{C}^{N}$. Since $\mathrm{C}^{N}$ is simply connected, there exists an entire function $b$ such that $f=P \cdot \exp b$. The result for other $\Delta_{r}$ can now be obtained by a change of variables.

2.8. Corollary. Assume that $f$ is an entire function in $\mathrm{C}^{N}(N \geq 1)$. If the slice functions $f_{w}$ bave the same finite number of zeros for each $w \in T^{N-1}$, then $f$ has the zeros of a polynomial.

Proof. This is an immediate consequence of Remark 1.4 and Theorem 2.1.

2.9. Corollary. Assume $f$ is an entire function in $\mathbf{C}^{N}(N \geq 2)$. If

(i) the slice function $f_{(1,1, \cdots, 1)}$ bas the zeros of a polynomial, and

(ii) $Z(f) \cap\left(r_{i} T^{N}\right)=\emptyset$ for some sequence of positive numbers $r_{i}>\infty$, then $Z(f) \cap \Delta$ is compact, and, therefore, $f$ has the zeros of a polynomial.

Proof. Set

$$
N_{r_{i}}(\alpha)=\frac{1}{2 \pi i} \int_{|\lambda|=r_{i}} \frac{f_{\alpha}^{\prime}(\lambda)}{f_{\alpha}(\lambda)} d \lambda, \quad \alpha \in T^{N-1},
$$

for $i=1,2, \cdots$. Then $N_{r_{i}}(\alpha)$ is the number of zeros of $f_{\alpha}(\cdot)$ whose modulus is less than $r_{i}$. Furthermore, since $N_{r_{i}}$ is continuous on $T^{N-1}$, it is constant. Set $N_{r_{i}}=N_{r_{i}}(\alpha), \alpha \in T^{N-1}$. Since $f_{(1,1, \cdots, 1)}$ has the zeros of a polynomial, there is a constant $M<\infty$ such that $N_{r_{i}}=N_{r_{j}}$ whenever $r_{i}, r_{j}>M$. Thus $Z(f) \cap\left(r T^{N}\right)$ $=\varnothing$, for all $r>M$. Therefore $Z(f)^{r_{i}} \cap \Delta^{r_{j}}$ is compact, and, by Theorem $2.1, f$ has the zeros of a polynomial.

3.

3.1. Theorem. Assume $f$ is an entire function in $\mathrm{C}^{N}(N \geq 2)$. Let $L$ be the 
union of all complex lines in $\Delta$ that lie in the zero set of any fixed entire function (not identically zero) in $\mathbf{C}^{N}$. If there is a variety $V$ in $\mathbf{C}^{N}$ of dimension $\leq N-2$ such that $(Z(f)-(L \cup V)) \cap \Delta$ is relatively compact in $\mathbf{C}^{N}$, then $f$ bus the zeros of a polynomial.

In Proposition 3.7 we will show that whenever $(Z(f)-(L \cup V)) \cap \Delta$ is relatively compact, $(Z(f)-L) \cap \Delta$ is also relatively compact, and it is shown in Lemma 3.8 that in this situation $f$ has the zeros of a polynomial. The presence of the variety $V$ in the statement of the theorem will allow us to obtain, in 3.5 and 3.6, two corollaries on the factorization of entire functions.

One might expect that the vanishing of an entire function on a complex line of $\Delta$ would imply that it had a homogeneous polynomial as one of its prime factors. This is the case in $\mathrm{C}^{2}$, but not in $\mathrm{C}^{3}$, as the following example will show.

3.2. Example. Consider the polynomial

$$
P\left(z_{1}, z_{2}, z_{3}\right)=4 z_{2} z_{3}+3 z_{1} z_{2}+z_{1} z_{3}+4 z_{1}+3 z_{3}+z_{2},
$$

and write

$$
P\left(\lambda, w_{1} \lambda, w_{2} \lambda\right)=\left(4 w_{1} w_{2}+3 w_{1}+w_{2}\right) \lambda\left\{\lambda+\left(4+3 w_{2}+w_{1}\right) /\left(4 w_{1} w_{2}+3 w_{1}+w_{2}\right)\right\}
$$

whenever $4 w_{1} w_{2}+3 w_{1}+w_{2} \neq 0$.

Both $4 w_{1} w_{2}+3 w_{1}+w_{2}$ and $4+3 w_{2}+w_{1} \cdot \operatorname{vanish}$ at $(-1,-1)$ and this is their only common zero. Furthermore,

$$
\left|\frac{4+3 w_{2}+w_{1}}{4 w_{1} w_{2}+3 w_{1}+w_{2}}\right|=\left|\frac{4+3 w_{2}+w_{1}}{w_{1} w_{2}\left(4+3 / w_{2}+1 / w_{1}\right)}\right|=1,
$$

whenever $\left(w_{1}, w_{2}\right) \in T^{2}$ and unequal to $(-1,-1)$; thus $P$ vanishes identically on $\{(\lambda,-\lambda,-\lambda): \lambda \in \mathbf{C}\}$, and its zeros have modulus one on every other complex line of $\Delta . P$ does not have a homogeneous factor, however, because there are no other complex lines on which it vanishes identically.

3.3. Remark. Let $L$ be the set of complex lines lying in $Z(b) \cap \Delta$, where $b$ is an entire function (not identically zero) in $C^{N}(N \geq 2)$; then $L$ is contained in the zero set of a homogeneous polynomial (not identically zero). If $f \equiv 0$ is an entire function in $C^{N}$ such that $(Z(f)-L) \cap \Delta$ is relatively compact, then the slice functions $f_{a}$ have the same finite number of zeros for each $a \in T^{N-1}$ for which $f_{a} \equiv 0$.

Proof. If $\sum_{i=n}^{\infty} b_{i}$ is the expansion of $b$ in terms of homogeneous polynomials with $b_{n} \neq 0$, then $L \subset Z\left(b_{n}\right)$.

When $N=2, L$ is the union of a finite number of complex lines and hence Remark 1.4 implies, in this situation, that $f_{a}$ has the same finite number of zeros for each $\alpha \in T$ with $f_{a} \equiv 0$. 
Assume the remark is true in $\mathrm{C}^{k}(2 \leq k<N)$. Then for each $\alpha \in T$, for which $f_{\alpha} \not 0$, there is an integer $m_{a}$ such that each slice function of $f_{a}$, that is not identically zero, has $m_{a}$ zeros. We will show that $m_{a}$ is independent of $\alpha_{\text {。 }}$ For each $\beta \in T$ define $g_{\beta}$ by setting $g_{\beta}(w)=f\left(w, \beta w_{1}\right), w=\left(w_{1}, \cdots, w_{N-1}\right) \epsilon$ $\mathrm{C}^{N-1}$, and define $n_{\beta}$ with respect to $g_{\beta}$ in the same way that $m_{\alpha}$ was defined for $f_{a}$.

Fix $a \in T$ such that $f_{a} \not \equiv 0$. For $N>3$ there is a $\gamma \in T^{N-3}$ such that $\left(f_{\alpha}\right)_{\gamma} \not \equiv 0$. Except for finitely many $\beta \in T$, therefore $\left(\left(f_{\alpha}\right)_{\gamma}\right)_{\beta} \not \equiv 0$ or when $N=3$, $\left(f_{a}\right)_{\beta} \neq 0$ 。 It follows, in either case, that $m_{a}=n_{\beta}$ for all but finitely many $\beta \in T$; therefore $m_{\alpha}=m_{\alpha^{\prime}}$ if $f_{\alpha^{\prime}} \not \equiv 0$ and $\alpha^{\prime} \in T$.

3.4. Corollary. Assume that $f \equiv 0$ is an entire function in $\mathrm{C}^{N}(N \geq 2)$, and let $L$ be the union of all complex lines in $\Delta$ lying in the zero set of some fixed entire function (not identically zero). Then $f$ has the zeros of a bomogeneous polynomial if and only if $(Z(f)-L) \cap \Delta$ contains no point other than the origin.

Proof. Necessity. If $Q$ is a homogeneous polynomial of degree $m$, then $Q_{w}(\lambda)=\lambda^{m} Q(1, w)$; thus for all $w \in T^{N-1}$, the slice functions $Q_{w}$, which are not identically zero, can have a zero only at the origin. writing

Sufficiency. By Theorem 3.1, $f$ has the zeros of a polynomial $Q$. Now

$$
Q_{w}(\lambda)=\sum_{j=0}^{m} b_{j}(1, w) \lambda^{j}, \quad w \in T^{N-1},
$$

where each $b_{j}$ is a homogeneous polynomial of degree $j$ and $b_{m} \not \equiv 0$, we see that $b_{j} \equiv 0$ for all $0 \leq j \leq m-1$ if $Q$ is not constant.

3.5. Corollary. Let $f, g$ be two entire functions (not identically zero) in $\mathrm{C}^{N}(N \geq 2)$, and let $L$ be the union of all complex lines in $\Delta$ that lie in the zero set of a fixed entire function (not identically zero). If $(Z(f)-(L \cup Z(g))) \cap \Delta$ is relatively compact, then each prime factor of $f$ is either also a prime factor of $g$, or has the zero of a polynomial. If, in addition, $f$ and $g$ are relatively prime, then $f$ has the zeros of a polynomial.

Proof. Let $p$ be a prime factor of $f$, and set $V=Z(p) \cap Z(g)$. Then $Z(p)-V=Z(p)-Z(g) \subset Z(f)-Z(g)$; therefore $(Z(p)-(L \cup V)) \cap \Delta$ is relatively compact. Now, if $p$ is not a prime factor of $g$, then $\operatorname{dim} V \leq N-2$, and, by Theorem 3.1, $p$ has the zeros of a polynomial。

If $f$ and $g$ are relatively prime, then $(Z(f)-(L \cup V)) \cap \Delta$ is relatively compact, where $V=Z(f) \cap Z(g)$ has dimension $\leq N-2$. Theorem 3.1 implies that $f$ has the zeros of a polynomial. 
3.6. Corollary. Assume $f \not \equiv 0$ and $g \equiv 0$ are entire functions in $\mathbf{C}^{N}(N \geq 2)$. If for each $w \in T^{N-1}$, the restriction $\left.\left(f_{w} / g_{w}\right)\right|_{|\lambda|>M}$ is bolomorpbic whenever $g_{w} \not \equiv 0$, then $P \cdot f=g \cdot b$, where $P$ is a polynomial and $b$ is entire. If, in addition, the restriction is zero free for all $w \in T^{N-1}$ for which $g_{w} \equiv 0$ and $f_{w} \not \equiv 0$, then there is a polynomial $Q$ and an entire function $k$ such that $P \cdot f=g \cdot Q \cdot \exp k$.

Proof. Entire functions $\tilde{f}, \tilde{g}$ can be chosen such that $f / g=\tilde{f} / \tilde{g}$ and $\operatorname{dim}(Z(\tilde{f}) \cap Z(\tilde{g})) \leq N-2$. Let $L$ be the union of all complex lines contained in $Z(f g) \cap \Delta$. The hypothesis implies that $(Z(\tilde{g})-(L \cup V)) \cap \Delta$ is relatively compact, where $V=Z(\tilde{f}) \cap Z(\tilde{g})$ has dimension $\leq N-2$. By Theorem 3.1, $\tilde{g}$ has the zeros of some polynomial $P$; hence the factorization $P \cdot f=g \cdot b$, where $b$ is entire.

The additional hypothesis implies that $(Z(\tilde{f})-(L \cup V)) \cap \Delta$ is relatively compact, and again Theorem 3.1 implies that $\tilde{f}$ has the zeros of some polynomial $Q$; hence $P \cdot f=g \cdot Q \cdot \exp k$ where $k$ is entire.

3.7. Proposition. Assume $f$ is an entire function in $\mathrm{C}^{N}(N \geq 2)$. Let $L$ be the union of all complex lines in $\Delta$ that lie in the zero set of any fixed entire function (not identically zero) in $\mathbf{C}^{N}$. If there is a variety $V$ in $\mathbf{C}^{N}$, of dimension $\leq N-2$, and a positive constant $M$ such that $(Z(f)-(L \cup V)) \cap \Delta$ $\subset M U^{N}$, then $(Z(f)-L) \cap \Delta \subset M U^{N}$, where $M U^{N}=\left\{z \in \mathbf{C}^{N}:\left|z_{i}\right|<M\right.$, $i=1, \cdots, N\}$.

Proof. It is enough to prove the proposition when $L$ is the union of all complex lines contained in $Z(f) \cap \Delta$; for by Remark 3.3 there is a homogeneous polynomial $Q$ such that $L \subset Z(Q) \cap \Delta$, and upon setting $\widetilde{L}=Z(Q) \cap \Delta$, we have that $(Z(f \cdot Q)-(\widetilde{L} \cup V)) \cap \Delta$ is relatively compact and $(Z(f)-L) \cap \Delta=$ $(Z(f \cdot Q)-\widetilde{L}) \cap \Delta$.

When $N=2, V$ has dimension 0 , and so it is a countable discrete subset of $\mathrm{C}^{2}$. Remark 1.4 then implies that $(Z(f)-L) \cap \Delta \subset M U^{N}$.

Assume that $N \geq 3$ and that the proposition is true in $\mathrm{C}^{K}, 2 \leq k<N$; we will show that it is true in $\mathbf{C}^{N}$. For $\alpha \in \mathbf{C}$, put

$$
E_{\alpha}^{i}=\left\{z \in \mathbf{C}^{N}: a z_{i}=z_{i+1}\right\}, \quad 1 \leq i \leq N-1,
$$

then

$$
\bigcap_{i=1}^{N-1} E_{a_{i}}^{i}=E_{\beta}=\left\{\left(\lambda, \beta_{1} \lambda, \cdots, \beta_{N-1} \lambda\right): \lambda \in \mathbf{C}\right\},
$$

where $\beta_{k}=\Pi_{i=1}^{k} \alpha_{i}, k=1, \cdots, N-1$. There is no loss of generality if we assume that $V$ does not contain any of the $N-2$ dimensional varieties 
$\left\{z \in \mathbf{C}^{N}: z_{i}=z_{k}=0\right\}$, where $i \neq k$ and $i, k=1, \cdots, N-1$, since each of these meets $\Delta$ only at the origin.

Fix any $i, 1 \leq i \leq N-1$. Then there are at most countably many $\alpha \in T$ such that $\operatorname{dim}\left(E_{\alpha}^{i} \cap V\right)=N-2$; for if the dimension is $N-2$, then $E_{a}^{i}$ contains one of the countably many $N-2$ dimensional components of $R(V)$, the set regular points of $V$, and, furthermore, if $\alpha \neq \alpha^{\prime}$, the only $N-2$ dimensional variety that $E_{a}^{i}$ and $E_{a^{\prime}}^{i}$ can share is

$$
E_{a}^{i} \cap E_{a^{\prime}}^{i}=\left\{z \in \mathbf{C}^{N}: z_{i}=z_{i+1}=0\right\}
$$

which by assumption is not one of the branches of $V$. On the other hand, if $\operatorname{dim}\left(E_{a}^{i} \cap V\right)<N-2$, then the inductive assumption can be applied to the restriction of $f$ to $E_{a^{\circ}}^{i}$ The set, therefore, of all $w \in T^{N-1}$ such that $f_{w} \not \equiv 0$ and its zeros are not bounded in modulus by $M$ is a countable subset of $T^{N-1}$; thus by Remark 1.4 it is empty.

3.8. Lemma. Assume that $f$ is an entire function in $\mathbf{C}^{N}(N \geq 2)$, and let $L$ be the complex lines in $\Delta$ that lie in the zero set of some fixed entire function (not identically zero). If $(Z(f)-L) \cap \Delta$ is relatively compact in $\mathrm{C}^{N}$, then $f$ has the zeros of a polynomial.

Proof. We will prove the lemma under the additional assumption that no homogeneous polynomial is a prime factor. This additional assumption introduces no loss of generality; for there are only finitely many such factors, since each vanishes at the origin, and if $f$ is expressed as the product of a homogeneous polynomial and an entire function $g$, then $g$ also satisfies the hypothesis of the lemma.

If $N=2, L$ is the union of a finite number of complex lines; hence Remark 1.4 implies that $Z(f) \cap \Delta$ is compact, and by Theorem $2.1 f$ has the zeros of a polynomial.

Assume $N \geq 3$ and that the lemma is true in $\mathrm{C}^{k}(2 \leq k<N)$; define $\tilde{f}$ by setting

$$
\tilde{f}(w, \alpha)=f_{a}(w), \quad w \in \mathbf{C}^{N-1}, a \in \mathbf{C} .
$$

Fix $\alpha^{\prime} \in T$. Since $\tilde{f}\left(\cdot, \alpha^{\prime}\right)$ has the zeros of a polynomial (not identically zero) in $\mathrm{C}^{N-1}$, there is a distinguished boundary $E^{N-2}$ and a constant $R>0$ such that $\tilde{f}_{\beta}\left(\lambda, a^{\prime}\right) \neq 0$, whenever $\beta \in E^{N-2}$ and $|\lambda| \geq R$. By continuity there is a closed disc $D$, centered at $\alpha^{\prime}$, such that $\tilde{f}_{\beta}(\lambda, \alpha) \neq 0$ whenever $\beta \in E^{N-2}$, $|\lambda|=R$ and $\alpha \in D$. The number of zeros of $\tilde{f}_{\beta}(\cdot, \alpha)$ with modulus less than $R$ is given by 


$$
N(\beta, \alpha)=\frac{1}{2 \pi i} \int_{|\lambda|=R} \frac{\mathscr{D}_{1} \tilde{f}_{\beta}(\lambda, \alpha)}{\tilde{f}_{\beta}(\lambda, \alpha)} d \lambda, \quad \beta \in E^{N-2}, \alpha \in D,
$$

where $\mathscr{D}_{1}$ denotes differentiation with respect to the first coordinate $\lambda . N$, being continuous and integer valued on the connected set $E^{N-2} \times D$, is constant; $N \equiv N\left(\beta, \alpha^{\prime}\right)=m$. Since $\tilde{f}_{\beta}(\cdot, \alpha)$ has at most $m$ zeros for each $\alpha \in H=T \cap D$ and each $\beta \in E^{N-2}$, it follows that $Z(\tilde{f}) \cap(\Delta d \times H)$ is compact. By Lemma 2.6 there are holomorphic functions $b_{j}$ in some neighborhood $\mathcal{O}$ of $\alpha^{\prime}$ in $\mathbf{C}$ such that, for each $a \in T \cap \mathcal{O}, \Lambda(\cdot, a)$, defined by

$$
\Lambda(w, \alpha)=\sum_{0 \leqslant|j|<n} b_{j}(\alpha) w^{j}, \quad w \in \mathbf{C}^{N-1},
$$

and $\tilde{f}(\cdot, \alpha)$ have the same zeros.

Select pairs $\Lambda_{i}, \Theta_{i}, i=1, \cdots, p$, as in the preceding paragraph, such that the neighborhoods $\mathcal{O}_{i}$ cover $T$. If $\alpha \in \mathcal{O}_{i} \cap \mathcal{\Theta}_{k} \cap T$, then $\Lambda_{i}(\cdot, \alpha)$ and $\Lambda_{k}(\cdot, a)$ are polynomials, not identically zero, whose quotient $\Lambda_{i}(\cdot, \alpha) / \Lambda_{k}(\cdot, \alpha)$ is a constant different from zero. Denote this number by $\tau_{i, k}(\alpha)$. It follows that the coefficients $b_{j}^{i}(\alpha)$, of $\Lambda_{i}(\cdot, \alpha)$, are just the corresponding coefficients $b_{j}^{k}(\alpha)$, of $\Lambda_{k}(\cdot, \alpha)$, multiplied by $\tau_{i, k}(\alpha)$; thus corresponding coefficients of $\Lambda_{i}(\cdot, \alpha)$ and $\Lambda_{k}(\cdot, \alpha)$ have the same zeros in some neighborhood of $\mathcal{O}_{i} \cap \mathcal{\Theta}_{k} \cap T$. Assume that $b_{J}^{i} \not \equiv 0$; then there is a function $H_{J}$, holomorphic in some neighborhood of $T$, which has the same zeros as each of the coefficients $b_{J}^{i}, i=1, \cdots, p$, in the common part of their domains, and $H_{J} \not \equiv 0$. Set

$$
H_{j}(\alpha)=\left(H_{J}(\alpha) / h_{J}^{i}(\alpha)\right) h_{j}^{i}(\alpha),
$$

for $\alpha$ in some neighborhood of $\mathcal{O}_{i} \cap T, 1 \leq i \leq p$ and $0 \leq|j| \leq n . H_{j}$ is well defined since

$$
\frac{H_{J}(\alpha)}{b_{J}^{i}(\alpha)} b_{j}^{i}(\alpha)=\frac{H_{J}(\alpha) \tau_{i k}(\alpha) b_{j}^{k}(\alpha)}{\tau_{i k}(\alpha) b_{J}^{k}(\alpha)}=\frac{H_{J}(\alpha) b_{j}^{k}(\alpha)}{b_{J}^{k}(\alpha)}
$$

therefore, each $H_{j}$ is holomorphic in some annular neighborhood $Q$ of $T$.

Set

$$
P(w, \alpha)=\sum_{0 \leqslant ! j \mid \leqslant n} H_{j}(\alpha) w^{j}, \quad w \in \mathbf{C}^{N-1}, \alpha \in Q .
$$

Then $P(\cdot, \alpha)$ has the same zeros as $\Lambda_{i}(\cdot, \alpha)$ for each $\alpha \in \mathcal{O}_{i} \cap T$ and each $i=1, \cdots, p$. In particular, $\tilde{f}(\cdot, \alpha)$ and $P(\cdot, \alpha)$ have the same zeros for all $\alpha \in T$; we will show that they have the same zeros for al! $\alpha$ in some neighborhood of $T$. The lemma then follows from Proposition 1.2 and Theorem 2.1. 
The Cartesian product $\mathrm{C}^{N-1} \times Q$ is a domain of holomorphy because each of the factors is a domain of holomorphy; furthermore, since $\mathrm{C}^{N-1} \times Q$ has the same homotopy type as the circle $S^{1}, H^{2}(Q \times \mathrm{C}, Z)$, the second cohomology group of $Q \times \mathbf{C}$ with integral coefficients, is isomorphic to $H^{2}\left(S^{1}, Z\right)=0$. In this situation there exists a pair of functions $g, b$ holomorphic in $\mathrm{C}^{N-1} \times Q$, and such that

(i) $\tilde{f} / P=g / h$, and

(ii) $\operatorname{dim}(Z(g) \cap Z(b))<N-1$;

see p. $25 i$ of [1]. Combined (i) and (ii) imply that

$$
Z(g) \subset Z(\tilde{f}) \text { and } Z(b) \subset Z(P) \text {. }
$$

For each $\alpha \in T, Z(g(\cdot, \alpha))=Z(b(\cdot, \alpha))$, because $Z(\tilde{f}(\cdot, \alpha))=Z(P(\cdot, \alpha))$; thus $V_{a}=Z(g(\cdot, \alpha)) \cap Z(b(\cdot, \alpha))$ is the zero set of both $g(\cdot, \alpha)$ and $b(\cdot, \alpha)$ whenever $a \in T$. If $V_{a} \neq \emptyset, a \in T$, then it contains one of the countably many irreducible branches of $Z(g) \cap Z(b)$; therefore, with the exception of at most a countable number of $\alpha \in T$, both $Z(g(\cdot, \alpha))$ and $Z(b(\cdot, \alpha))$ are empty.

Fix $\beta \in T^{N-2}$ for which $P_{\beta} \not \equiv 0$. In the following argument we will show that, for all $\alpha \in T, b_{\beta}(\cdot, \alpha)$ is zero free except for at most a countable number of values of $\alpha$ where $b_{\beta}(\cdot, \alpha) \equiv 0 . P_{\beta}$ can be expressed as

$$
P_{\beta}(\lambda, \alpha)=\sum_{j=0}^{m} \psi_{j}(\alpha) \lambda^{j}, \quad(\lambda, \alpha) \in \mathbf{C} \times Q,
$$

where the coefficients are holomorphic and $\psi_{m} \not \equiv 0$. Then from (1) and (2), the zeros of $b_{\beta}(\cdot, \alpha)$ are bounded uniformly in modulus for all $\alpha$ in any relatively compact subset of the connected set $Q-Z\left(\psi_{m}\right)$; therefore, by Remark 1.4 $b_{\beta}(\cdot, a)$ has the same number of zeros for each $\alpha \in Q-Z\left(\psi_{m}\right)$. As a result of the preceding paragraph $Q-Z\left(\psi_{m}\right)$ contains values $\alpha$ for which $b_{\beta}(\cdot, \alpha)$ has no zeros; therefore $b_{\beta}(\cdot, \alpha)$ is zero free for all $\alpha \in Q-Z\left(\psi_{m}\right)$. In order, therefore, for $b_{\beta}(\cdot, \alpha)$ to have a zero, it is necessary, because $Z\left(\psi_{m}\right)$ is discrete, that $b_{\beta}(\cdot, a) \equiv 0$. Therefore for each fixed $\alpha \in Q$, Corollary 3.4 implies that $b(\cdot, a)$ has the zeros of a homogeneous polynomial provided $P(\cdot, \alpha) \equiv 0$.

If $Z(b(\cdot, a)) \neq \varnothing$, then $b(0, a)=0$; thus the set of $a$ in $Q$ such that $Z(b(\cdot, a)) \neq \emptyset$ is discrete. Now fix $\alpha_{0} \in Q$ such that $b\left(\cdot, \alpha_{0}\right) \equiv 0$, and choose a distinguished boundary $E^{N-2} \subset \mathrm{C}^{N-2}$ such that the zeros of $b_{\beta}\left(\cdot, a_{0}\right)$ are bounded uniformly in modulus for all $\beta \in E^{N-2}$. Since the points $\alpha \in Q$ for which $Z(b(\cdot, a)) \neq \varnothing$ are discrete, it follows from Remark 1.4 that $Z\left(b\left(\cdot, \alpha_{0}\right)\right)$ $=\emptyset$. Therefore $Z(b(\cdot, a))=\emptyset$ for all $a \in T$, and by continuity it follows that $Z(b(\cdot, a))=\varnothing$ for all $a$ in some neighborhood of $T$.

The same argument can be carried out for $g$ if (2) is replaced by a similar 
expression obtained from the polynomial which has the same zeros as the restriction of $f$ to $\left\{\left(\lambda, \tau, \beta_{1} \lambda, \cdots, \beta_{N-2} \lambda\right):(\lambda, \tau) \in \mathrm{C}^{2}\right\}$. This concludes the proof.

3.9. Proof of Theorem 3.1. By Proposition 3.7, $(Z(f)-L) \cap \Delta$ is relatively compact; therefore Lemma 3.8 implies that $f$ has the zeros of a polynomial.

\section{REFERENCES}

1. R. C. Gunning and H. Rossi, Analytic functions of several complex variables, Prentice-Hall, Englewood Cliffs, N. J., 1965. MR 31 \#4927.

2. W. Rudin, A geometric criterion for algebraic varieties, J. Math. Mech. 17 (1967/68), 671-683. MR 36 \#2829.

DEPARTMENT OF MATHEMATICS, UNIVERSITY OF KENTUCKY, LEXINGTON, KENTUCKY 40506 